\title{
Irradiation d'une grossesse méconnue : revue à court terme de 17 cas
}

\author{
N. CHAHED*, A. MHIRI*, S. GAIGI*, S. MTIMET*
}

(Manuscrit reçu le 27 août 1999, révisé le 30 mai 2000, accepté le 9 juillet 2000)

RÉSUMÉ Nous avons revu et étudié 47 dossiers de femmes enceintes, ignorant leur grossesse et ayant eu une ou plusieurs explorations radiologiques. Nous avons distingué deux groupes. Le premier groupe G1 est constitué de 12 femmes irradiées à l'étage sous diaphragmatique durant la phase critique d'organogenèse, à des doses gonadiques estimées entre 12 et $52 \mathrm{mGy}$, et pour lesquelles une interruption de la grossesse a été indiquée. Le second groupe G2 est formé de 35 femmes irradiées soit indirectement, soit directement à l'étage sus diaphragmatique, à des doses n'excédant pas $10 \mathrm{mGy}$ et pour lesquelles la poursuite de la grossesse a été préconisée. Nous avons mené une enquête auprès de ces femmes, pour étudier les issues de leur grossesse. Seules 17 femmes ont pu être revues, 7 de G1 et 10 de G2. L'analyse des issues relève l'absence d'avortement spontané, d'anomalies majeures et d'affections malignes. Chez les enfants revus, nous avons noté la présence de $\mathbf{4}$ anomalies mineures : une discrète dysmorphie faciale, une atrésie membraneuse de l'anus, un hypospadias, et un bec de lièvre. Le nombre restreint de cas étudiés ne permet pas d'établir une corrélation certaine entre les anomalies et l'irradiation. Il semble au contraire que les anomalies observées relèvent du risque malformatif général, en raison des très faibles doses reçues par le produit de conception et de son âge.

ABSTRACT Irradiation of unknown pregnancy: review of 17 cases.

The article summarizes the analysis of 47 cases of pregnant women who did not know their pregnancy and had undergone one or more diagnostic radiological examinations. We distinguished two groups. The first group G1 consisting of 12 women whose exposure to radiation had been focused under diaphragmatic region and occurred during the critical period of organogenesis with a gonadal dose estimated to range 12 to $52 \mathrm{mGy}$. A therapeutic abortion had been recommended to these women. The second group $\mathrm{G} 2$ consisting of 35 women whose exposure to radiation interested directly or indirectly the above diaphragmatic region with dose lower than $10 \mathrm{mGy}$. To these women, a recommendation to continue their pregnancy had been given. We investigated only 17 pregnant women issues; 7 from G1 et 10 from G2. We noted that there were no spontaneous abortion or major abnormalities or malignant diseases. However, 4 minor abnormalities were identified among the examined children sample: facial dysmorphism, anus atresia, hypospadias and harelip. These anomalies could not be attributed to the irradiation effects because the conceptus received very low doses during the minimal risk gestation period. We believe that the malformations observed may be attributed to congenital malformation risk.

* Centre national de radioprotection, Hôpital d'enfants, 1006 Bab Souika, Tunis, Tunisie. Tél : 2I6-577 776 : Fax : 216-57। 697. 


\section{Introduction}

La présence de risque embryo-fœtal lié à une irradiation externe au cours de la grossesse a fait l'objet de plusieurs travaux expérimentaux (Baskar et Devi, 1996 ; Berlin, 1996 ; Bouhnik et al., 1988; Kimler et al., 1994 ; ICRP Publication 60, 1991 ; Dygalo et al., 1997) et d'études de données cliniques et épidémiologiques humaines (Elefant et al., 1991; Vidal et al., 1993; Greffet, 1994; Hoshino et al., 1997; Jacquet et al. 1995 ; Gisserot, 1981 ; Loffredo et al., 1977 ; Mole, 1991, 1992). Il est cependant difficile de chiffrer ce risque, et de là est née la divergence des opinions.

Aux faibles doses utilisées en radiodiagnostic, pour certains auteurs il n'y a pratiquement pas de risque surajouté. Pour d'autres il existe un risque certain, ce qui peut justifier une interruption thérapeutique de grossesse, sans qu'il soit possible de déterminer toutes ses conséquences, en particulier à long terme.

Aussi, nous sommes-nous proposés d'étudier ce risque à partir des issues de grossesses de femmes involontairement exposées au cours d'examens radiologiques. Cette étude permettra d'aider le médecin à décider d'une conduite à tenir: soit rassurer la mère quant à la poursuite de sa grossesse, soit au contraire proposer une interruption thérapeutique de la grossesse.

\section{Matériel et méthodes}

Nous avons revu 47 dossiers du Centre national de radioprotection (CNRP). Ils concernaient des femmes enceintes, dont la grossesse était méconnue lorsqu'elles ont été exposées aux rayons $\mathrm{X}$ au cours d'un examen radiologique.

Ces femmes ont été adressées au CNRP, entre mars 1992 et janvier 1997, pour avis sur la conduite à tenir.

Les paramètres intervenant dans l'avis proposé par le CNRP étaient, la dose gonadique, le terme de la grossesse au moment de l'irradiation et au moment la consultation au CNRP, et dans quelques cas: la pathologie associée, l'âge de la patiente, les antécédents médicaux et l'état psychologique du couple.

La dose seuil retenue par le CNRP pour conseiller une interruption de grossesse a été fixée à 10 mGy et ce en s'inspirant de la méthode de Magnin, citée par Gisserot (1981).

L'ordre de grandeur de la dose délivrée à l'embryon ou au foetus a été estimée à partir des données de la Société française des physiciens des hôpitaux (Roux et Harvath, 1979). 
Nous avons étudié les dossiers en distinguant deux groupes de femmes: - un premier groupe G1 constitué de 12 femmes auxquelles une interruption thérapeutique de grossesse a été proposée;

- et un second groupe G2 composé de 35 femmes auxquelles la poursuite de la grossesse a été préconisée.

Nous avons mené une enquête auprès de ces femmes pour étudier les issues de leur grossesse. Seules 17 femmes ont pu être revues :

- 7 femmes du groupe G1, pour lesquelles nous avons compté sur les résultats des examens embryologiques et caryotypiques des débris de l'évacuation utérine pour confirmer le bien-fondé de l'attitude prise par le CNRP.

- 10 femmes du groupe G2, pour lesquelles nous nous sommes intéressés à la fois au suivi de la grossesse, (éventuelle complication induite par les effets des rayonnements ionisants) et à son issue: examen néonatalogique des nouveaux-nés et suivi pédiatrique en vue d'apprécier les développements staturo-pondéral et psychomoteur.

\section{Résultats}

Nous présentons l'analyse de l'ensemble des dossiers des groupes G1 et G2 ainsi que les observations faites sur les issues de grossesse des femmes que nous avons revues.

3.1. Douze femmes du groupe G1 ont subi une irradiation durant la période de radiosensibilité maximale comprise entre la $3^{\mathrm{c}}$ et la $10^{\mathrm{e}}$ semaine d'aménorrhée (S.A.). - Sept d'entre elles ont été irradiées à l'étage sous diaphragmatique, région particulièrement sensible pour l'embryon et/ou le fœtus. Elles totalisent 16 examens radiologiques : 3 d'entre elles ont eu un examen; 2 ont eu deux examens; 1 a eu trois examens et 1 en a eu six. Ces différentes irradiations se répartissent en 3 examens d'abdomen sans préparation (ASP), 2 urographies intra veineuses (UIV), 2 phlébographies des membres inférieurs, 2 hystérographies, 2 radiographies du bas$\sin , 2$ radiographies du rachis dorso-lombaire, 1 scanner du rachis lombaire et une radiographie du thorax. Les doses gonadiques reçues par ces femmes sont toutes supérieures à $10 \mathrm{mGy}$ et varient entre 12 et $52 \mathrm{mGy}$ selon les cas.

- Quatre femmes ont eu des examens radiologiques de l'étage sus-diaphragmatique (mammographie, pneumoscopie et scopie lors d'un cathétérisme). La dose gonadique estimée étant nettement inférieure à $10 \mathrm{mGy}$, l'interruption de la grossesse a été indiquée pour des raisons autres que radiologiques, que nous avons eu à traiter en collaboration avec des collègues d'autres spécialités.

- Une seule femme a été exposée indirectement, lorsqu'elle immobilisait son enfant au cours d'examens radiologiques. En raison de la difficulté de reproduire les conditions réelles de l'examen radiologique, la dose reçue n'a pas pu être évaluée. 
TABLEAU I

Issues des femmes du groupe G1 qui ont été revues et auxquelles une interruption de grossesse avait été conseillée.

G1 group issues of examined women to whom a therapeutic abortion had been recommended.

\begin{tabular}{|c|c|c|c|c|}
\hline $\begin{array}{l}\text { Nature } \\
\text { de l'examen }\end{array}$ & $\begin{array}{c}\text { Date } \\
\text { de l'irradiation }\end{array}$ & $\begin{array}{c}\text { Dose gonadique } \\
\text { estimée }\end{array}$ & Indication & Résultat des issues \\
\hline $\begin{array}{l}\text { A.S.P } \\
+ \text { U.I.V }\end{array}$ & $\begin{array}{c}3,10 \text { et } 11 \text { S.A. } \\
12 \text { S.A. }\end{array}$ & $23 \mathrm{mGy}$ & suivie & fœetus non récupéré \\
\hline Pneumoscopie & 3S.A. & non évalué & suivie & foetus non récupéré \\
\hline Mammographies & 4 S.A. & $0,16 \mathrm{mGy}$ & suivie & foetus non récupéré \\
\hline Hystérographie & 8 S.A. & $12 \mathrm{mGy}$ & suivie & foetus non récupéré \\
\hline $\begin{array}{l}\text { Phlébographie } \\
\text { des membres inférieurs }\end{array}$ & 4 et 6 S.A. & $18 \mathrm{mGy}$ & non suivie & enfant normal ( $l^{\text {er }}$ cas) \\
\hline Hystérographie & 5 S.A. & $12 \mathrm{mGy}$ & non suivie & $\begin{array}{c}\text { enfant }+ \text { discrète } \\
\text { dysmorphie faciale } \\
\left(2^{\mathrm{e}} \text { cas }\right)\end{array}$ \\
\hline $\begin{array}{l}\text { Bassin + thorax } \\
+ \text { scanner dorsolombaire } \\
\text { Rachis dorsolombaire }\end{array}$ & $\begin{array}{c}9 \text { S.A. } \\
22 \text { S.A. + } 15 \text { S.A. }\end{array}$ & $52 \mathrm{mGy}$ & non suivie & enfant normal ( $\left.3^{\mathrm{e}} \mathrm{cas}\right)$ \\
\hline
\end{tabular}

3.2. L'étude des 7 issues des grossesses de G1 (Tab. I) permet de relever :

- l'absence d'avortement spontané;

- la pratique de 4 avortements thérapeutiques par aspiration ou par curetage. Malheureusement, aucun fœtus n'a été récupéré et aucun examen fotopathologique n'a été pratiqué;

- la naissance de 3 enfants pour 3 femmes qui n'ont pas tenu compte de l'avis du CNRP et qui ont laissé évoluer leur grossesse.

Le $1^{\text {er }}$ cas est né à terme, tout à fait normal. Revu à l'âge de 4 ans, il présente un bon développement staturo-pondéral et psychomoteur. L'enfant a été exposé in utéro lors d'une phlébographie et d'un cathétérisme pratiqués chez la mère entre la $4^{\mathrm{e}}$ et la $6^{\mathrm{e}} \mathrm{S}$.A. La dose a été estimée à $18 \mathrm{mGy}$.

Le $2^{\mathrm{e}}$ cas a présenté une macrosomie à la naissance. Revu à l'âge de 20 mois, l'examen clinique est normal, malgré la présence d'une discrète dysmorphie faciale avec un hypertélorisme, des «traits grossiers» à la limite de la normale et un âge osseux normal. L'enfant est né d'une femme ayant eu une hystérographie à la $5^{\mathrm{e}} \mathrm{S}$.A. La dose a été évaluée à $12 \mathrm{mGy}$.

Pour le dernier cas, l'accouchement programmé a eu lieu par césarienne à la $36^{\mathrm{e}} \mathrm{S}$.A. en rapport avec une présentation dystocique. L'enfant est né sans anomalies morphologiques évidentes et présente à sa revue à 13 mois, un développement staturo-pondéral et psychomoteur normal. Il s'agit d'un enfant né d'une 
TABLEAU II

Issues des femmes du groupe G2 qui ont été revues et auxquelles une poursuite de la grossesse a été prescite.

G2 issues of group examined women to whom a recommendation to continue their pregnancy had been indicated.

\begin{tabular}{|c|c|c|c|c|}
\hline $\begin{array}{l}\text { Nature } \\
\text { de l'examen }\end{array}$ & $\begin{array}{c}\text { Date } \\
\text { de l'irradiation }\end{array}$ & $\begin{array}{l}\text { Dose gonadique } \\
\text { estimée }\end{array}$ & Indication & Résultat des issues \\
\hline Thorax ( 2 clichés) & 5 S.A. & $0,2 \mathrm{mGy}$ & non suivie & fœetus non récupéré \\
\hline Thorax & IS.A. & $0,1 \mathrm{mGy}$ & suivie & enfant vivant normal \\
\hline Thorax & $5 \mathrm{~S} . \mathrm{A}$. & $0,1 \mathrm{mGy}$ & suivie & $\begin{array}{l}\text { enfant vivant: } \\
\text { atrésie de l'anus }\end{array}$ \\
\hline Pneumoscopie & 10 S.A. & non évaluée & suivie & enfant vivant normal \\
\hline Pneumoscopie & 5S.A. & non évaluée & suivie & enfant vivant normal \\
\hline Rachis cervical + dorsal & 4 S.A. & $0,13 \mathrm{mGy}$ & suivie & $\begin{array}{l}\text { enfant vivant : } \\
\text { bec de lièvre }\end{array}$ \\
\hline Cheville & 10 S.A. & $<1 \mathrm{mGy}$ & suivie & enfant vivant normal \\
\hline $\begin{array}{l}\text { Irradiation indirecte } \\
\text { (cavum + panoramique } \\
\text { dentaire) }\end{array}$ & 4-6 S.A. & non évaluée & suivie & enfant vivant normal \\
\hline $\begin{array}{l}\text { Irradiation indirecte } \\
\text { (thorax) }\end{array}$ & 4 S.A. & non évaluée & suivie & enfant vivant normal \\
\hline $\begin{array}{l}\text { Irradiation indirecte } \\
\text { (thorax) }\end{array}$ & 2 S.A. & non évaluée & suivie & $\begin{array}{l}\text { enfant vivant : } \\
\text { hypospadias }\end{array}$ \\
\hline
\end{tabular}

femme âgée de 41 ans et hospitalisée dans un service d'orthopédie pour traumatisme de la charnière dorso-lombaire, entraînant une paraplégie. Elle a reçu plusieurs clichés radiologiques irradiant l'utérus pendant la fin de la période d'organogenèse, alors que sa grossesse était ignorée. La décision d'interrompre la grossesse a été refusée par les gynécologues pour raison réglementaire. La dose a été estimée à 52 mGy.

3.3. Les 35 femmes du groupe $\mathrm{G} 2$ ont été exposées à une irradiation directe et indirecte, avec une dose gonadique faible, n'excédant pas les $10 \mathrm{mGy}$. Pour la plupart, le contenu utérin n'a pas été pris dans le faisceau direct des rayons X. La période gestationnelle au moment de l'irradiation correspond :

- aux phases de gamétogenèse ou de préimplantation, pour 8 cas ;

- à la période fœtale, située au-delà de la $12^{\mathrm{e}} \mathrm{S}$.A., pour 5 femmes ;

- à la période d'organogenèse, pour 22 femmes. Durant cette période, le risque de malformations est maximal pour l'embryon. Cependant, pour la majorité d'entre elles (10 femmes), il s'agit d'une irradiation indirecte, liée à leur présence dans la salle de radiologie pour immobiliser leur enfant, ou d'une irradiation directe intéressant l'étage sus diaphragmatique ( 8 femmes ont eu un examen du thorax). Seules 4 femmes ont subi des examens de l'étage sous-diaphragmatique: A.S.P. (2), cheville (1), bassin (antéro postérieur) (1) et rachis lombo-sacré (1). 
3.4. L'étude des issues de grossesse des 10 femmes de G2 indique (Tab. II) :

- l'absence d'avortement spontané;

- un avortement thérapeutique pratiqué chez une femme ayant eu une radioscopie du thorax à la $5^{\mathrm{e}} \mathrm{S}$.A. L'interruption de la grossesse réalisée à la $10^{\mathrm{e}} \mathrm{S}$.A. a été indiquée pour raison de pharmaco-vigilance. L'examen anatomo-pathologique n'a pu être effectué ;

- la naissance de 9 enfants, dont les mères ont eu une grossesse et un accouchement normaux. Ces enfants ont un poids, une taille et un périmètre crânien conformes aux valeurs «standards» ainsi qu'un bon développement psychomoteur. Néanmoins, trois enfants présentent des anomalies : une atrésie membraneuse de l'anus, un hypospadias et un bec de lièvre.

\section{Discussion et conclusion}

La pratique d'un examen radiologique chez une femme dont la grossesse est méconnue est une situation à laquelle le médecin est souvent confronté. Plusieurs questions se posent :

- Y-a-t-il risque létal in utéro, risque d'avortement spontané ou accroissement de la tératogenèse?

- Faut il prescrire une interruption de la grossesse ou sa poursuite?

Les risques sont en rapport direct avec la dose reçue par le foetus ou l'embryon et avec son âge au moment de l'irradiation. Aussi, le médecin doit-il, non seulement évaluer rétrospectivement la dose fœtale ou embryonnaire, mais également tenir compte des éléments suivants, qui constituent des critères importants dans son appréciation.

1. L'avortement spontané au cours de la grossesse est fréquent. Dans un service de maternité d'un centre hospitalier de Tunis, 70 cas d'avortement spontané ont été dénombrés pour 1355 accouchements, soit 5,16\%. Si un avortement spontané survient chez une femme enceinte irradiée, il est difficile de l'imputer formellement aux effets de l'irradiation.

2. Toute grossesse comporte un risque d'anomalie. Quatre à $6 \%$ des nouveaux-nés sont porteurs d'une anomalie congénitale. En Tunisie, la fréquence de malformation congénitale est estimée à $3 \%$. L'accroissement de ce pourcentage induit par l'irradiation est faible et difficile à mettre en évidence (Yamazaki et Schull, 1990).

3. Les femmes traitées pour stérilité constituent un cas particulier où plusieurs facteurs interviennent : l'âge de la femme, l'interférence des examens pratiqués, surtout l'hystérographie, avec une grossesse non encore diagnostiquée.

4. L'action tératogène des rayonnements ionisants diminue quand la dose est d'autant plus faible et/ou fractionnée (Tubiana, 1979; Uma Devi et Baskar, 1996; Vergouven et al., 1995; WHO, 1996). Pour ce qui est de la dose seuil, dose audessous de laquelle aucun effet aussi mineur soit-il n'est engendré chez aucun 
individu, il n'y a toujours pas de réponse. Certains auteurs considèrent qu'une dose, aussi faible soit-elle, peut entraîner des malformations, alors que d'autres pensent que le risque n'existe qu'à partir d'une dose seuil mais dont la valeur ne fait pas l'unanimité. Pour les doses d'exposition induites par le radiodiagnostic et inférieures à $50 \mathrm{mGy}$, aucun effet n'a été constaté (Yamazaki et Schull, 1990). Toutefois, la susceptibilité génétique de chaque individu vis-à-vis d'un facteur tératogène mérite d'être prise en considération (Zamman et al., 1992).

5. L'âge gestationnel au moment de l'irradiation est également important à considérer. Toutes les études confirment la loi du tout ou rien pendant la période de préimplantation: il y a soit avortement spontané, soit poursuite de la grossesse avec une croissance normale de l'embryon.

Durant l'organogenèse, les risques d'anomalies graves sont importants alors qu'ils deviennent négligeables au cours de la période fotale, et ce au fur et à mesure que la grossesse évolue jusqu'à son terme.

Plusieurs auteurs ont proposé des règles de conduite à tenir, en se basant sur la dose et l'âge gestationnel au moment de l'irradiation. Ces deux paramètres constituent les critères primordiaux sur lesquels repose l'évaluation du risque radique.

S'il est vrai que le nombre de cas étudiés ne constitue pas un échantillon représentatif, les résultats obtenus apportent néanmoins une contribution modeste à ce problème complexe.

En plus des difficultés d'ordre général liées à ce sujet, telles que la détermination de la dose embryonnaire ou fœtale, il y a celles qui sont particulières à notre étude. Il s'agit du nombre important de femmes non reexaminées, de la non-récupération du fotus et de la non-réalisation de son examen fotopathologique.

Les études consacrées à ce sujet soulignent que lors d'un avortement thérapeutique, l'examen embryologique est toujours difficile à réaliser car la méthode aspiratoire endommage l'embryon et ne permet donc pas une étude complète.

Toutefois, notre étude permet de relever l'absence:

- d'avortement spontané chez toutes les femmes que nous avons revues;

- d'anomalies morphologiques et/ou fonctionnelles majeures, notamment la microcéphalie et le retard mental. Nous avons constaté un bon éveil psychomoteur, le quotient intellectuel n'a pas été évalué;

- d'affection maligne. Il y a lieu de noter cependant que le délai entre l'irradiation et la date de l'examen pédiatrique est court. Ce genre de pathologie (leucémie et cancer) est réputée avoir un temps de latence très long;

- d'anomalies même chez l'enfant le plus irradié. L'âge avancé de la mère (41 ans) aurait été un facteur additif de tératogenèse s'il y avait eu anomalie. 
TABLEAU III

Modèle de conduite à proposer en cas d'irradiation involontaire d'une femme enceinte.

Proposal of a behaviour model for irradiated pregnant women.

\begin{tabular}{|c|c|c|}
\hline $\begin{array}{c}\text { Dose gonadique } \\
\text { estimée }\end{array}$ & $\begin{array}{l}\text { Áge gestationnel } \\
\text { au moment de l'irradiation }\end{array}$ & $\begin{array}{l}\text { Interruption thérapeutique } \\
\text { de la grossesse }\end{array}$ \\
\hline Dose $<10 \mathrm{mGy}$ & - quel que soit l'âge & - pas de légitimité \\
\hline $\begin{array}{l}\text { Dose entre } 10 \\
\text { et } 50 \mathrm{mGy}\end{array}$ & $\begin{array}{l}\text { - gamétogenèse } \\
\text { ou préimplantation } \\
\text { - organogenèse ( } 3 \text { à } 10 \text { S.A.) } \\
\text { - période foetale > } 12 \text { S.A. }\end{array}$ & $\begin{array}{l}\text { - pas de légitimité } \\
\text { - à accepter } \\
\text { - pas de légitimité, } \\
\text { mais à surveiller }\end{array}$ \\
\hline Dose $>50 \mathrm{mGy}$ & - quel que soit l'âge & - à accepter \\
\hline
\end{tabular}

Chez les enfants que nous avons revus, nous avons observé la présence de quatre anomalies.

Dans le groupe G1, la discrète dysmorphie faciale avec hypertélorisme décelée chez un enfant est une anomalie morphologique minime, pouvant être considérée comme banale, à caractère familial ou en rapport avec une aberration chromosomique liée à l'irradiation.

Dans le groupe G2, les trois malformations peuvent être considérées comme «coincidentales ». Pour ces 3 femmes, d'une part l'embryon n'a pas été exposé directement et, d'autre part, les doses gonadiques estimées sont faibles. Elles sont inférieures à $10 \mathrm{mGy}$ et nettement au-dessous de $100 \mathrm{mGy}$, seuil estimé pour l'espèce humaine par la Commission internationale de protection radiologique, dans le cas où le risque résulterait d'un effet déterministe (ICRP, 1991). En France, l'Académie des sciences recommande l'interruption de la grossesse au-delà de 200 mGy. Entre 100 et 200 mGy, la conduite à tenir est discutée cas par cas. Au-dessous de 100 mGy le risque attribuable à l'irradiation n'est pas retenu.

Ainsi, la présence de ces anomalies ne pourrait être attribué uniquement à l'effet de l'irradiation et elles entreraient plutôt dans le risque malformatif général.

Cette évaluation, aussi approximative soit-elle, permet de proposer le modèle présenté dans le tableau III.

Ce travail devrait donner lieu à des développements futurs, aussi bien sur le plan individuel (tel que l'information des femmes à radiographier et sur l'intérêt de déclarer leur éventuelle grossesse), que sur le plan épidémiologique. 


\section{IRRADIATION D'UNE GROSSESSE MÉCONNUE}

\section{RÉFÉRENCES}

Baskar R., Devi P.U. ( 1996) Long term effect of prenatal exposure to low level of gamma radiation on neurophysiology of mouse, Indian J. Exp. Biol. 34, 887-890.

Berlin L. (1996) Radiation exposure and the pregnant patient, Am. J. Radiol. 167, 1377-1379.

Bouhnik H., Bard J.J., Chavaudra J. et al. (1988) Société française des physiciens d'hôpital (SFPH) Commission radiodiagnostic. Évaluation des doses délivrées au cours des examens radiologiques. J. Radioprot. 23, 1-37.

Kimler B.F., Vidal-Pergola G.M., Perterson S.L., LI X., Norton S. (1994) Effect of in utero radiation dose fractionation on rat post natal development, behavior and brain structure; 3 hour interval, Neurotoxicology 15, 183-190.

ICRP Publication 60 (1991) Recommendations of the International Commission on Radiological Protection, Ann. ICRP 21 (1-3), 22.

Dygalo N.N., Sakharov D.G., Shishkura G.T. (1997) Corticosterone and testosterone in blood of adult rats: the effects of low doses and the times of the action of ionizing radiation during intrauterine development, Radiat. Biol. Radioecol. 37 (3) 377-381.

Elefant E., Boyer M., Nizard S. (1991) Rayonnements ionisants et tératovigilance, J. Radiol. 72 (12) $681-684$.

Vidal-Pergola G.M., Kimler B.F., Norton S. (1993) Effect of in utero irradiation on the post-natal development behavior, and brain structure of rats: dose fractionation with a 6 h interval, Radiat. Res. 134, 369-374.

Grellet J. (1994) Irradiation du foetus et de l'enfant en radiodiagnostic, J. Radiol. 75, 213-216.

Hoshino K., Kameyama Y., Inouye M. (1997) Split-dose effect of X-irradiation on the induction of cell death in the fetal mouse brain, J. Radiat. Res. 32, 23-27.

Jacquet P., de Saint-Georges L., Vankerkom J., Baugnet-Mahieu L. (1995) Embryonic death, dwarfism and foetal malformations after irradiation of embryos at the zygote stage: studies on two mouse strains, Mut. Res. 332, 73-87.

Gisserot (1981) Médecine et Armées, 9, 5, 399-406.

Loffredo V., Vibert M.R., Merger R. (1977) Essai d'évaluation du risque fœtal après radiographie abdomino-pelvienne pratiquée en début de grossesse, Rev. Française Gynécol. 72, 91-98.

Mole R.H. (1991) Post-script 1991 : Radiosensiblité in utero. Effets cancerigenes, Radiologie 72, 707-708.

Mole R.H. (1992) ICRP and impairment of mental function following prenatal irradiation, J. Radiol. Prot. 12, 93-105.

Roux C.H., Harvath C. (1979) Tératologie, Encycl. Med. Paris, Obstétrique A10, 51 I6.

Steven R.H., Li X., Kimler B.F. (1993) Is there an adaptive response to radiation in the developing brain of the fetal rat? Radiat. Res. 136, 293-296.

Tubiana M. (1979) Problèmes posés par l'irradiation des femmes enceintes. Effets des radiations ionisantes sur l'embryon et le fotus, Bull. Cancer 1, 156-164.

Uma Devi P., Baskar R. (1996) Influence of gestational age at exposure on the prenatal effects of gamma radiation, Int. J. Radiat. Biol. 70, 45-52

Vergouven R.P.F.A., Huiskamp R., Bas R.J. et al. (1995) Radiosensitivity of testicular cells in the fetal mouse, Radiat. Res. 141, 66-73.

WHO (1996) Rapport sur les conséquences sanitaires de l'accident de Tchernobyl, pp. 2-32.

Yamazaki J.N., Shull W.J. (1990) Prenatal loss and neurological abnormalities among children of the atomic bomb, JAMA 264, 605-609

Zamman M.S., Hupp E.W., Lancaster F.E. (1992) Brain myelination in rats treated with ionizing radiation in utero, Environ. Sci. Health 27, 621-638.

Zamman M.S., Hupp E.W., Lancaster F.E. (1993) Locomotion and physical development in rats treated with ionising radiation in utero, Envimon. Sci. Health 28, 105-125. 